\title{
Vanishing Thermal Equilibration for Hole-Conjugate Fractional Quantum Hall States in Graphene
}

\author{
Saurabh Kumar Srivastav $\odot,{ }^{1}$ Ravi Kumar, ${ }^{1}$ Christian Spånslätt $\odot,{ }^{2,3,4}$ K. Watanabe, ${ }^{5}$ T. Taniguchi, ${ }^{5}$ \\ Alexander D. Mirlin, ${ }^{3,4,6,7}$ Yuval Gefen, ${ }^{3,8}$ and Anindya Das $\mathbb{1}^{1, *}$ \\ ${ }^{1}$ Department of Physics, Indian Institute of Science, Bangalore 560012, India \\ ${ }^{2}$ Department of Microtechnology and Nanoscience (MC2), Chalmers University of Technology, S-412 96 Göteborg, Sweden \\ ${ }^{3}$ Institute for Quantum Materials and Technologies, Karlsruhe Institute of Technology, 76021 Karlsruhe, Germany \\ ${ }^{4}$ Institut für Theorie der Kondensierten Materie, Karlsruhe Institute of Technology, 76128 Karlsruhe, Germany \\ ${ }^{5}$ National Institute of Material Science, 1-1 Namiki, Tsukuba 305-0044, Japan \\ ${ }^{6}$ Petersburg Nuclear Physics Institute, 188300 St. Petersburg, Russia \\ ${ }^{7}$ L. D. Landau Institute for Theoretical Physics RAS, 119334 Moscow, Russia \\ ${ }^{8}$ Department of Condensed Matter Physics, Weizmann Institute of Science, Rehovot 76100, Israel
}

(Received 21 December 2020; accepted 20 April 2021; published 27 May 2021)

\begin{abstract}
Transport through edge channels is responsible for conduction in quantum Hall (QH) phases. Robust quantized values of charge and thermal conductances dictated by bulk topology appear when equilibration processes become dominant. We report on measurements of electrical and thermal conductances of integer and fractional QH phases, realized in hexagonal boron nitride encapsulated graphite-gated bilayer graphene devices for both electron and hole doped sides with different valley and orbital symmetries. Remarkably, for complex edges at filling factors $\nu=\frac{5}{3}$ and $\frac{8}{3}$, closely related to the paradigmatic hole-conjugate $\nu=\frac{2}{3}$ phase, we find quantized thermal conductance whose values $\left(3 \kappa_{0} T\right.$ and $4 \kappa_{0} T$, respectively where $\kappa_{0} T$ is the thermal conductance quantum) are markedly inconsistent with the values dictated by topology $\left(1 \kappa_{0} T\right.$ and $2 \kappa_{0} T$, respectively). The measured thermal conductance values remain insensitive to different symmetries, suggesting its universal nature. Our findings are supported by a theoretical analysis, which indicates that, whereas electrical equilibration at the edge is established over a finite length scale, the thermal equilibration length diverges for strong electrostatic interaction. Our results elucidate the subtle nature of crossover from coherent, mesoscopic to topology-dominated transport.
\end{abstract}

DOI: 10.1103/PhysRevLett.126.216803

According to the bulk-edge correspondence principle [1-3], certain characteristics of gapless edge modes are constrained by the topological order in the gapped bulk. This turns out to be a subtle issue for hole-conjugate fractional quantum Hall $(\mathrm{FQH})$ phases, whose edges are complex, i.e., hosting counterpropagating modes: $n_{d}$ moving downstream (the direction defined by semiclassical skipping orbits at the edge) and $n_{u}$ moving upstream (opposite to downstream). The quantized two-terminal electrical conductance for these states has been predicted to be $G=\nu\left(e^{2} / h\right)$, while the thermal conductance is $G_{Q}=\left|n_{d}-n_{u}\right| \kappa_{0} T$. Here, $\nu$ is the bulk Landau-level filling factor, $\kappa_{0}=\left[\left(\pi^{2} k_{B}^{2}\right) / 3 h\right], k_{B}$ is he Boltzmann constant, $h$ is the Planck constant, and $T$ is the temperature [4-6]. Observing the quantization of $G$ and $G_{Q}$ requires full equilibration of the counterpropagating edge modes $[7,8]$.

The paradigmatic example of a complex edge occurs at $\nu=\frac{2}{3}$ and consists of counterpropagating 1 (downstream) and $\frac{1}{3}$ (upstream) modes [9]. In the presence of disorder and strong electrostatic intermode interaction (parametrized by a single parameter $\Delta \rightarrow 1$ ), these bare modes renormalize to one ballistic downstream charge mode with $G=$ $\frac{2}{3}\left(e^{2} / h\right)$ and one ballistic upstream neutral mode only at the low-temperature $(T \rightarrow 0)$ and infinite-edge-length $(L \rightarrow \infty)$ limit [10]. However, for finite length and at finite temperature, a robust $G=\frac{2}{3}\left(e^{2} / h\right)$ requires full equilibration among the bare modes leading to incoherent transport $[7,8,11]$. In the opposite limit of coherent, nonequilibrated edge transport, one finds [7] $G=\frac{4}{3}\left(e^{2} / h\right)$. Experimental observation of the crossover from $G=\frac{4}{3}\left(e^{2} / h\right)$ (entirely nonequilibrated) to $G=\frac{2}{3}\left(e^{2} / h\right)$ (fully equilibrated) has so far been reported only in a GaAs/AlGaAs-based device [12]. The corresponding crossover length scale $\ell_{\mathrm{eq}}^{C}$ defines the electrical equilibration length. Likewise, the thermal equilibration length $\ell_{\mathrm{eq}}^{H}$ defines the crossover from a thermally nonequilibrated edge with thermal conductance $G_{Q}=\left(n_{d}+n_{u}\right) \kappa_{0} T$ to the topologically constrained and equilibrated thermal conductance $G_{Q}=\left|n_{d}-n_{u}\right| \kappa_{0} T$. For $\nu=\frac{2}{3}\left(\right.$ where $\left.n_{d}=n_{u}=1\right)$ and its cousin states, $\nu=\frac{5}{3}$ and $\frac{8}{3}$, this topology-dictated $G_{Q} / \kappa_{0} T$ is 0,1 , and 2 , respectively. For $\nu=\frac{2}{3}$, the zero value is expected with increasing 
$L$ as $G_{Q} \sim \ell_{\mathrm{eq}}^{H} / L$, signaling heat diffusion [7,8]. To date, experimental studies of thermal transport on complex $\mathrm{FQH}$ edges have been performed only in GaAs/AlGaAs-based structures [5,6], including $\nu=\frac{2}{3}$, and yielded values of $G_{Q}$ consistent with the equilibrated regime. However, similar to the electrical conductance, an experimental manifestation of the dichotomy between equilibrated and nonequilibrated values of heat conductance on complex $\mathrm{FQH}$ edges is missing. In this context, a different system like bilayer graphene (BLG) with more degrees of freedom (valley and orbital) together with unprecedented universal edge profile $[13,14]$, due to atomically sharp confining potential, are ideal platforms to study the thermal transport. For electronlike $\mathrm{FQH}$ edges in this system (with only downstream modes), topology-dictated and universal thermal conductance values were found [15], but no measurements have so far been performed for complex hole-conjugate $\mathrm{FQH}$ edges.

Here, we report measurements of the thermal and electric conductance of a variety of QH phases, realized in $h$-BN encapsulated graphite-gated BLG devices, for both electron and hole doping, using sensitive noise thermometry $[5,6,15,16]$, where all the symmetries (spin, valley, and orbitals) are broken [17-19]. For integer $\mathrm{QH}(\nu=1,2,3,4)$ and electronlike $\mathrm{FQH}$ states $\left(\nu=\frac{4}{3}, \frac{7}{3}\right)$ we obtain the expected values for $G\left(1\left(e^{2} / h\right), 2\left(e^{2} / h\right), 3\left(e^{2} / h\right)\right.$, $4\left(e^{2} / h\right), \frac{4}{3}\left(e^{2} / h\right), \frac{7}{3}\left(e^{2} / h\right)$, respectively) and $G_{Q}$ (within accuracy of $5 \%, 1 \kappa_{0} T, 2 \kappa_{0} T, 3 \kappa_{0} T, 4 \kappa_{0} T, 2 \kappa_{0} T, 3 \kappa_{0} T$, respectively). For the hole-conjugate phases $\nu=\frac{5}{3}$ and $\frac{8}{3}, G$ shows expected values $\left(\frac{5}{3}\left(e^{2} / h\right)\right.$ and $\frac{8}{3}\left(e^{2} / h\right)$, respectively), corresponding to electrically equilibrated edges. At the same time, and most remarkably, $G_{Q}$ is found to be $3 \kappa_{0} T$ and $4 \kappa_{0} T$, respectively, corresponding to thermally nonequilibrated edges. Our results of thermal conductance on $\mathrm{FQH}$ states for different valleys and orbitals further suggest a universality: a topology-dictated $G_{Q}$ for electronlike FQH states $\left(\frac{4}{3}, \frac{7}{3}\right)$, but entirely nonequilibrated $G_{Q}$ for hole-conjugate $\mathrm{FQH}$ states $\left(\frac{5}{3}, \frac{8}{3}\right)$. To explain the striking contrast between electric and thermal equilibration for hole-conjugate $\mathrm{FQH}$ states, we present a theoretical analysis of edge equilibration in the strong interaction limit [33]. In the limit of $\Delta \rightarrow 1$ we find that, while $\ell_{\mathrm{eq}}^{C}$ remains finite, $\ell_{\mathrm{eq}}^{H}$ diverges as $1 /(\Delta-1)$, indicating vanishing thermal equilibration. This gives rise to a new regime $\ell_{\text {eq }}^{C} \ll L \ll$ $\ell_{\text {eq }}^{H}$ observed here; in Fig. 1(a) we contrast it to the regime of fully equilibrated transport, $\ell_{\mathrm{eq}}^{C} ; \ell_{\mathrm{eq}}^{H} \ll L$.

For the thermal conductance measurement, we used two bottom graphite-gated devices ( $D 1$ and $D 2)$, where the graphene was encapsulated between two $h$-BN layers, each with thickness of $\sim 20 \mathrm{~nm}$. The device fabrication is described in the Supplemental Material [19]. Similar to our previous work [15], our devices consist of a floating metallic reservoir in the middle, connected to edge channels on both sides, as shown schematically in Fig. 1(b) [15]. The distances from the floating contact to the transverse contacts and cold grounds in Fig. 1(b) were $\sim 3 \mu \mathrm{m}$ $(4 \mu \mathrm{m})$ and $\sim 6 \mu \mathrm{m}(8 \mu \mathrm{m})$ for $D 1(D 2)$, respectively (see [19] for optical images). The electrical conductance was measured using a standard lock-in technique, whereas the thermal conductance was measured with noise thermometry $[5,6,15,16,19]$. In Fig. 1(c), the blue curve represents $G_{S}\left(I_{S} / V_{S}\right)$ measured at the source contact $(S)$ for the $D 1$ device as a function of the bottom graphite gate voltage $\left(V_{\mathrm{BG}}\right)$.

Plateaus appear at $\nu=\frac{5}{3}, \frac{7}{3}$, and $\frac{8}{3}$ along with the integer $\mathrm{QH}$ plateaus at $\nu=1,2$, and 3. Similarly, for the $D 2$ device, plateaus appear at $\nu=\frac{4}{3}, \frac{7}{3}$, and $\frac{8}{3}$ ([19] Fig. S6). In Fig. 1(c), the red (black) curve shows the measured resistance $R_{T}=V_{T} / I_{S}\left(R_{R}=V_{R} / I_{S}\right)$ at the $T(R)$ contact along the transmitted (reflected) path from the floating contact $(D 1)$. Measured resistances along these paths are exactly half of the resistance measured at the $S$ contact, which strongly suggests that the injected current is equally divided from the floating contact to both sides of the graphene channel. The resistance values at the $S, R$, and $T$ contacts for $\nu=\frac{5}{3}$ and $\frac{8}{3}$ are consistent with the charge equilibration of the bare modes along the propagation length. To further confirm the charge equilibration, we measure the conventional two-probe electrical conductance of $\nu=\frac{2}{3}$ in another device (D3 with $L \sim 5-6 \mu \mathrm{m}$ ) with three- and four-probe configurations. In Fig. 1(d), the quantized value is fixed at $\sim 39 \mathrm{k} \Omega$. By contrast, if there was no charge equilibration, the resistance values obtained using the Landauer-Büttiker model [37] for our devices will be quite different [19].

In order to measure the thermal conductance, a dc $\left(I_{S}\right)$, injected at the $S$ contact [Fig. 1(b)], flows toward the floating reservoir and the outgoing current splits into two equal parts to the cold grounds. The power dissipation at the floating reservoir due to joule heating is $J_{Q}=$ $\left(I_{S}^{2} / 4 \nu G_{0}\right)[15,19]$, and thus the electrons in the floating reservoir will be heated to a new steady-state temperature $\left(T_{M}\right)$, determined by the following heat balance relation $[5,6,15,16,38,39]$ :

$$
\begin{gathered}
J_{Q}=J_{Q}^{e}\left(T_{M}, T_{0}\right)+J_{Q}^{e-\mathrm{ph}}\left(T_{M}, T_{0}\right), \\
J_{Q}=0.5 N \kappa_{0}\left(T_{M}^{2}-T_{0}^{2}\right)+J_{Q}^{e-\mathrm{ph}}\left(T_{M}, T_{0}\right) .
\end{gathered}
$$

Here, $J_{Q}^{e}\left(T_{M}, T_{0}\right)$ is the electronic contribution of the heat current via $N$ chiral edge modes, and $J_{Q}^{e-p h}\left(T_{M}, T_{0}\right)$ is the heat loss via electron-phonon coupling. The $T_{M}$ is obtained by measuring the excess thermal noise; $S_{I}=\nu k_{B}\left(T_{M}-\right.$ $\left.T_{0}\right) G_{0}[5,6,15,16,38,40-42]$, along the outgoing edge channels as shown in Fig. 1(b). Figure 2(a) shows the measured excess thermal noise $S_{I}$ as a function of current $I_{S}$ for $\nu=1$ (red), 2 (black), and 3 (blue) (D1). The noise and current axes of Fig. 2(a) are converted to $J_{Q}$ and $T_{M}$ and 
(a)

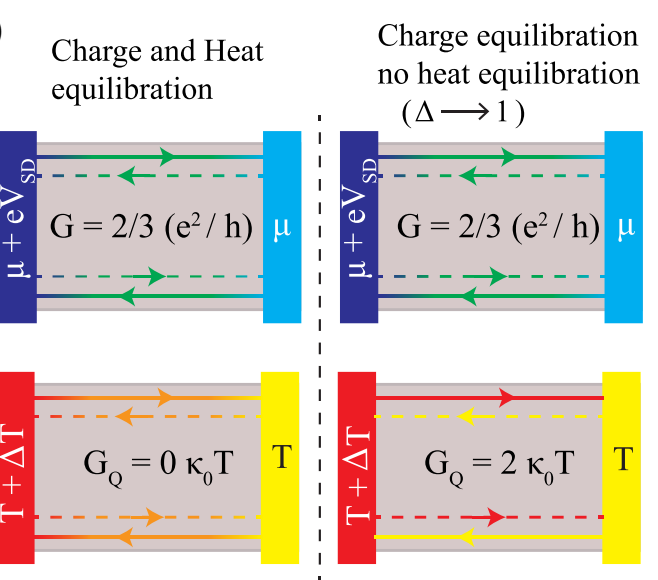

(b)

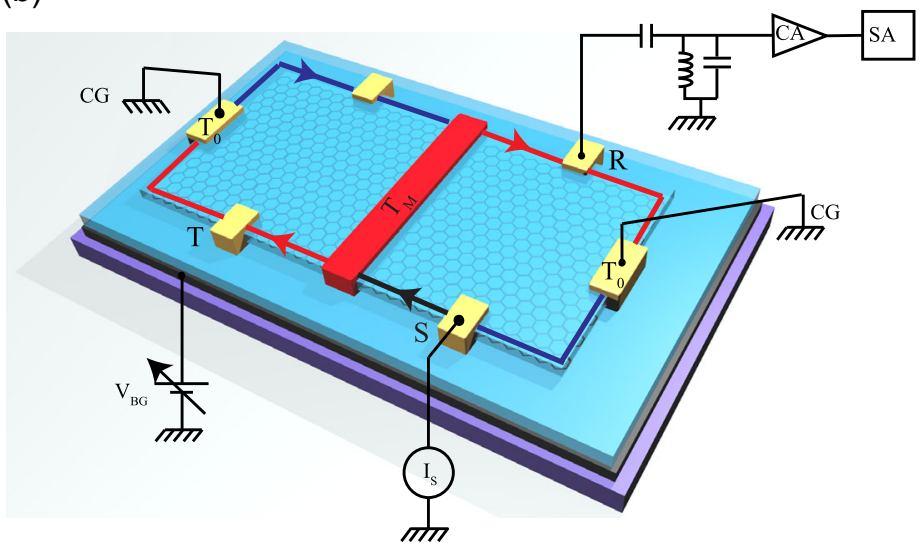

(c)

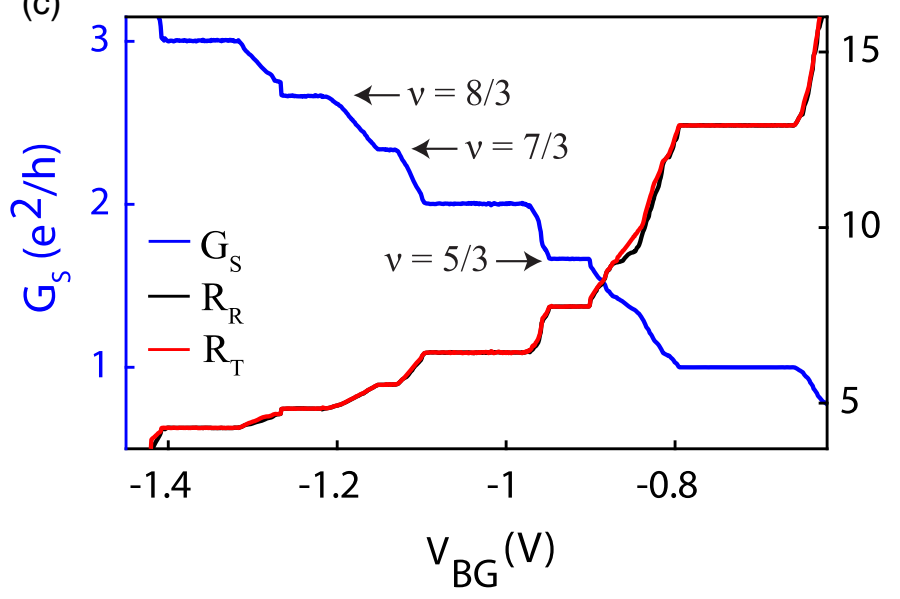

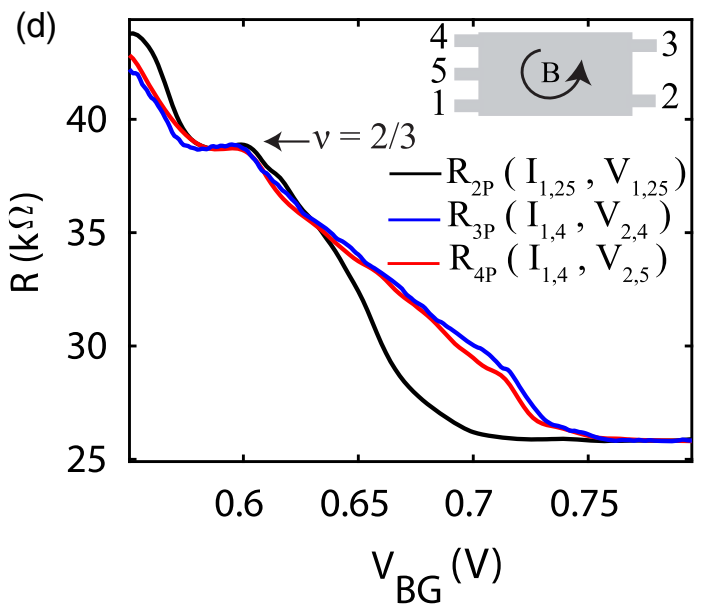

FIG. 1. Equilibration, device schematic, and QH response. (a) Left: voltage (top) and temperature (bottom) profile in colors with changing intensity along the edge for $L \gg \ell_{\mathrm{eq}}^{C} ; \ell_{\mathrm{eq}}^{H}$. In this limit, $G$ is $\frac{2}{3}\left(e^{2} / h\right)$ and $G_{Q}$ goes to zero diffusively. Right: voltage and temperature profile in limit $\ell_{\mathrm{eq}}^{C} \ll L \ll \ell_{\mathrm{eq}}^{H}$ realized at $\Delta \rightarrow 1$. While $G$ is still $\frac{2}{3}\left(e^{2} / h\right)$, one has now $G_{Q}=2 \kappa_{0} T$. Solid and dashed lines correspond to the down- and upstream eigenmodes, respectively. (b) Schematic of device with measurement setup. The device is set in the integer quantum Hall $(\mathrm{QH})$ regime at $\nu=1$. An injected current $I_{S}$ (black line) is absorbed in the floating reservoir (red contact) and terminates into two cold grounds. The electrical and thermal conductances are measured at low $(228 \mathrm{~Hz})$ and high frequency $(\sim 758 \mathrm{kHz}$ with a $L C R$ resonant circuit), respectively. (c) The blue line is the $G_{S}\left(I_{S} / V_{S}\right)$ as a function of $V_{\mathrm{BG}}$ at $B=10 \mathrm{~T}$ for the $D 1$ device. The red and black lines are the measured resistances (right $y$ axis) at the $T$ and $R$ contacts, respectively. The robust fractional plateaus at $\frac{5}{3}\left(e^{2} / h\right), \frac{7}{3}\left(e^{2} / h\right), \frac{8}{3}\left(e^{2} / h\right)$ with weaker plateau $\sim \frac{4}{3}\left(e^{2} / h\right)$ clearly visible. (d) The conductance measured in two- (black), three- (blue), and four-probe (red) configurations are plotted for the $D 3$ device. The inset show the contact positions. The first number in the subscript of $I$ corresponds to current fed contact and the remaining numbers label grounded contacts. The same notation is used for voltage $(V)$ measurements.

plotted in Fig. 2(b). To extract $G_{Q}$ for each filling factor, we have plotted $J_{Q}$ as a function of $T_{M}^{2}-T_{0}^{2}$ in Fig. 2(c). The solid circles represent the experimental data, while the solid lines are the linear fits of $G_{Q}$ with $0.99,1.96$, and $3.01 \kappa_{0} T$ for $\nu=1,2$, and 3 , respectively. Similarly, for device $D 2$, $G_{Q}$ was found to be $\sim 0.99,2.05,3.04$, and $3.96 \kappa_{0} T$ for $\nu=1,2,3$, and 4, respectively ([19] Fig. S7), which shows an excellent match with its expected theoretical values. Note that $J^{e-\mathrm{ph}}$ was negligible up to $T_{M} \sim 60 \mathrm{mK}$, and also, the heat Coulomb blockade [43] was expected to be absent for our graphite-gated devices [19].

Figure 3(a) shows $S_{I}$ as a function of $I_{S}$ for $\nu=\frac{5}{3}$ (red), $\frac{7}{3}$ (black), and $\frac{8}{3}$ (blue) for $D 1$. Experimental curves for $7 / 3$ and $8 / 3$ are shifted vertically by $1 \times 10^{-29}$ and $3 \times$ $10^{-29} A^{2} / \mathrm{Hz}$ for clarity. From these raw data, the $T_{M}$ was extracted as a function of $J_{Q}$ [19]. In Fig. 3(b), $J_{Q}$ is plotted as a function of $T_{M}^{2}-T_{0}^{2}$ as shown by the colored circles, and the solid lines are the theoretical curves for $G_{Q}=1 \kappa_{0} T$ (magenta), $2 \kappa_{0} T$ (brown), $3 \kappa_{0} T$ (red), and $4 \kappa_{0} T$ (blue). The linear fittings to the measured data in Fig. 3(b) gives $G_{Q} \sim 3.03,2.96$, and $4.03 \kappa_{0} T$ for $\frac{5}{3}, \frac{7}{3}$, and $\frac{8}{3}$, respectively. Similarly, $G_{Q} \sim 1.96,3.01$, and $3.94 \kappa_{0} T$ for $\frac{4}{3}, \frac{7}{3}$, and $\frac{8}{3}$, respectively, for device $D 2$ ([19] Fig. S8). For the particlelike states $\frac{4}{3}$ and $\frac{7}{3}$, the measured value of $G_{Q}$ is in excellent agreement with the expected 
(a)

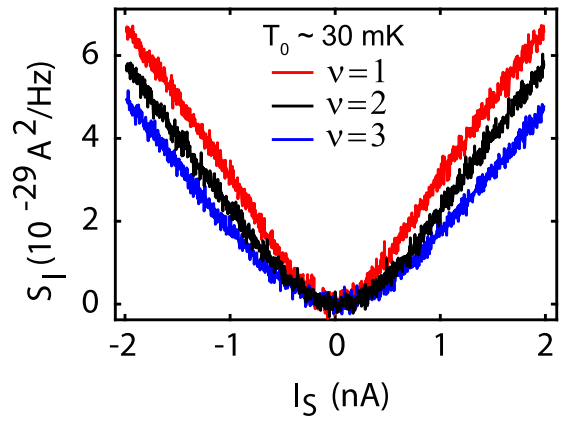

(b)

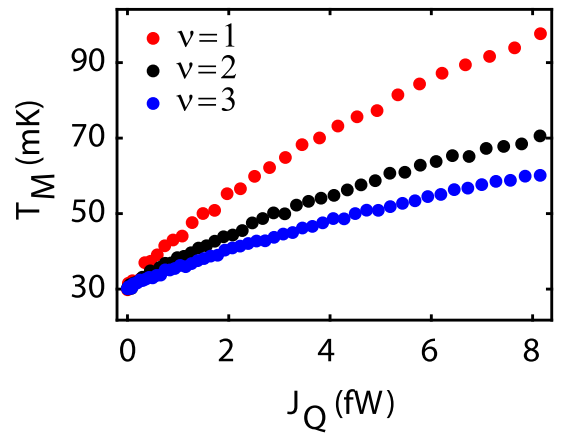

(c)

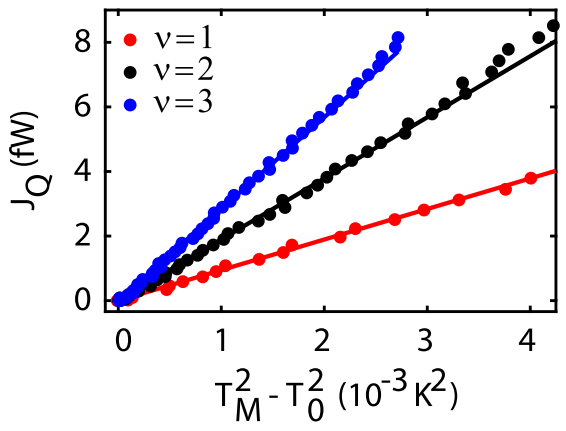

FIG. 2. Thermal conductance for integer QH states. (a) Excess thermal noise $S_{I}$ as a function of source current $I_{S}$ at $\nu=1$ (red), 2 (black), and 3 (blue). (b) The temperature $T_{M}$ of the floating contact as a function of the dissipated power $J_{Q}$ for $\nu=1$ (red), 2 (black), and 3 (blue), respectively. (c) $J_{Q}$ (solid circles) as a function of $T_{M}^{2}-T_{0}^{2}$ for $\nu=1$ (red), 2 (black), and 3 (blue), respectively. Solid lines are linear fits with $G_{Q}=0.99,1.96$, and $3.01 \kappa_{0} T$ for $\nu=1-3$, respectively.

theoretical values. However, for the holelike FQH states $\frac{5}{3}$ and $\frac{8}{3}$, the measured $G_{Q}$ strikingly matches with $\left(n_{d}+\right.$ $\left.n_{u}\right) \kappa_{0} T$ rather than the expected topological quantum number of $\left|n_{d}-n_{u}\right| \kappa_{0} T=1 \kappa_{0} T$ and $2 \kappa_{0} T$, respectively. In Fig. 3(c), we plot $\lambda=\Delta J_{Q} /\left(0.5 \kappa_{0}\right)$ as a function of $T_{M}^{2}$ for two different configurations of $\Delta \nu=\frac{5}{3}-1$ (red) and $\frac{8}{3}-2$ (black) to extract the contribution of the partially filled Landau level with $\nu=\frac{2}{3}$ out of the data for $\frac{5}{3}$ and $\frac{8}{3}$. Linear fits give $2.02 \kappa_{0} T$ and $2.06 \kappa_{0} T$, respectively, for $G_{Q}$ of the $\nu=\frac{2}{3}$ state. For the $D 2$ device, the fit yields $1.99 \kappa_{0} T$ ([19] Fig. S8). It is worth mentioning here that the thermal conductance of $\frac{7}{3}$ and $\frac{8}{3}$ states observed in the hole doped (device D1) and electron doped (device D2) regime are the same, irrespective of the different orbital nature of their wave functions, which is $N=1(0)$ in the hole (electron) doped regime [17-19]. In fact, the extracted thermal conductance of the $\frac{2}{3}$-like state from $\frac{5}{3}$ and $\frac{8}{3}$ [Fig. 3(c)] is also the same, irrespective of the different orbital nature of wave functions, which is $N=1(0)$ for $\frac{5}{3}$ $\left(\frac{8}{3}\right)$. This establishes the universality of our results.

The observed values of the thermal conductance for $\nu=\frac{5}{3}$ and $\frac{8}{3}$ imply essentially vanishing thermal equilibration between counterpropagating modes. To explain this, we consider a model of counterpropagating 1 and $\frac{1}{3}$ modes in the uppermost Landau level. In the presence of interchannel interactions, this level consists of two emergent, counterpropagating eigenmodes. Their dimensionless charge conductances are $g_{ \pm}=(\Delta \pm 1) / 3$. Importantly, their dimensionless heat conductances are unity, independent of $\Delta$. Tunneling facilitated by random disorder leads to equilibration between these modes. Calculating the charge and heat tunneling currents, we derive thermal $\left(\ell_{\mathrm{eq}}^{H}\right)$ and charge $\left(\ell_{\mathrm{eq}}^{C}\right)$ equilibration lengths [19] (a)

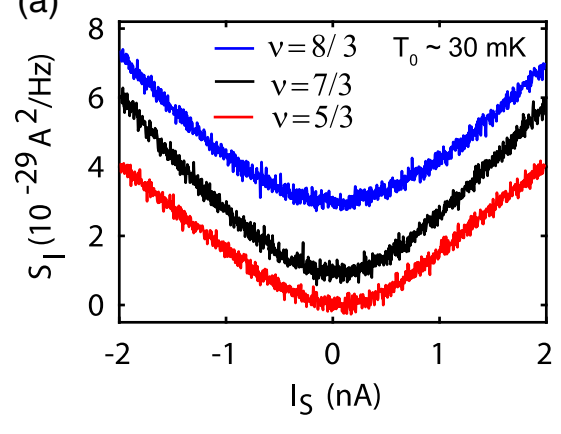

(b)

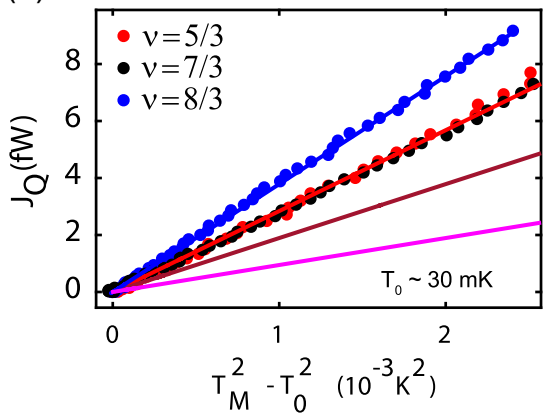

(c)

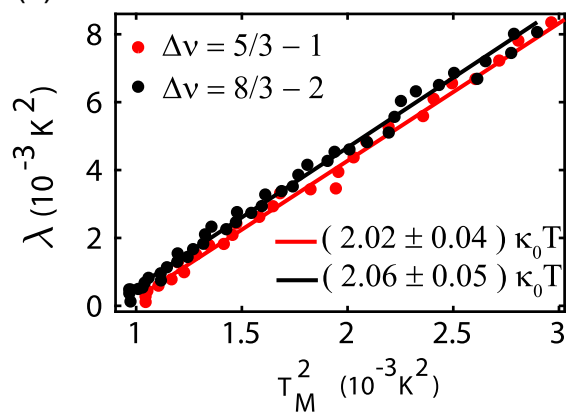

FIG. 3. Thermal conductance for fractional QH states. (a) $S_{I}$ as a function of $I_{S}$ at $\nu=5 / 3$ (red), 7/3 (black), and $8 / 3$ (blue). Experimental curves for $7 / 3$ and $8 / 3$ are shifted vertically by $1 \times 10^{-29}$ and $3 \times 10^{-29} A^{2} / \mathrm{Hz}$. (b) $J_{Q}$ (solid circles) as a function of $T_{M}^{2}-T_{0}^{2}$ for $\nu=5 / 3$ (red), $7 / 3$ (black), and 8/3 (blue). The solid magenta, brown, red, and blue lines represent $G_{Q}=1 \kappa_{0} T, 2 \kappa_{0} T$, $3 \kappa_{0} T$, and $4 \kappa_{0} T$, respectively. The linear fits of the solid circles give $G_{Q}=3.03,2.96$, and $4.03 \kappa_{0} T$ for $\nu=5 / 3,7 / 3$, and $8 / 3$, respectively. (c) $\lambda=\Delta J_{Q} /\left(0.5 \kappa_{0}\right)$ as a function of $T_{M}^{2}$ for $\Delta \nu=5 / 3-1$ (red) and $\Delta \nu=8 / 3-2$ (black), where $\Delta J_{Q}=J_{Q}\left(\nu_{i}, T_{M}\right)-J_{Q}\left(\nu_{j}, T_{M}\right)$. Solid lines represent linear fits. Extracted values of $G_{Q}$ of the $2 / 3$-like $\mathrm{FQH}$ states are $2.02 \kappa_{0} T$ and $2.06 \kappa_{0} T$ for $\Delta \nu=5 / 3-1$ and $\Delta \nu=8 / 3-2$, respectively. 


$$
\ell_{\mathrm{eq}}^{H / C} \propto \mathcal{C}^{H / C}(\Delta) T^{2-2 \Delta}, \quad \mathcal{C}^{H}(\Delta) \sim \frac{1}{\Delta-1}, \quad \mathcal{C}^{C}(\Delta) \sim 1,
$$

where we have displayed only the dependence on the temperature $T$ and $\Delta$. Our key observation is that the coefficient $\mathcal{C}^{H}(\Delta)$ diverges for $\Delta \rightarrow 1$, implying a very large $\ell_{\mathrm{eq}}^{H}$. This happens because the tunneling current between eigenmodes is proportional to $\Delta-1$. The region $\Delta$ close to 1 corresponds to very strong interactions. We argue that the sharp confining potential of our graphene devices, where the screening graphite gate is separated from the electron gas of graphene by a thin insulating $h$-BN layer $(\sim 10-20 \mathrm{~nm})$ $[13,14,44,45]$, favors this regime in contrast to the shallow confining potential in GaAs/AlGaAs devices $[5,6]$. For $\ell_{\text {eq }}^{C}$, the smallness of the tunneling current is compensated by the smallness of the charge conductance of one of the eigenmodes $\left(g_{-}\right)$. The eigenmode conductances determine the effect of tunneled charge on the local voltages. Tunneling of a finite charge to the "almost neutral" chiral mode results in an enhanced effect on the local voltage of the mode, facilitating easier equilibration of the local chemical potentials. Technically, this will compensate the $\Delta-1$ factor of the tunneling current, leading to $\mathcal{C}^{C}(\Delta) \sim 1$ (the same result for $\ell_{\text {eq }}^{C}$ is also obtained [46] by explicitly considering electrostatics of fractionalization-renormalized tunneling.) As a result, for $\Delta$ close to $1, \ell_{\mathrm{eq}}^{C} \ll \ell_{\mathrm{eq}}^{H}$, which creates a broad regime of system sizes $\ell_{\mathrm{eq}}^{C} \ll L \ll \ell_{\mathrm{eq}}^{H}$, thereby explaining the experimental observations of efficient charge equilibration but vanishing thermal equilibration.

In conclusion, the findings of this Letter are a remarkable manifestation of a transport regime with partial equilibration: the charge transport is in an equilibrated regime, while the heat transport is nonequilibrated irrespective of the different symmetry nature of wave functions. Both quantities, in the asymptotic limits of an equilibrated-nonequilibrated edge, respectively, are determined by the edge quantum numbers. We expect that such regimes should be relevant also to other FQH states and materials. In fact, several proposed mechanisms for explaining the observed heat conductance $\frac{5}{2} \kappa_{0} T$ at $\nu=5 / 2$ involve patterns of partial equilibration within the (non-Abelian) anti-Pfaffian state [47-50]. We envisage future work exploring the influence of partial equilibration on noise, decoherence, and FQH interferometry.

Useful discussions with I. V. Gornyi, D. G. Polyakov, and I. V. Protopopov on the theory part of this work are acknowledged. A. D. thanks the Department of Science and Technology (DST), India for financial support (DSTO2051) and acknowledges support from the Swarnajayanti Fellowship of the DST/SJF/PSA-03/2018-19. A. D. also thanks Moty Heiblum and Jainendra Jain for useful discussions. S. K. S. acknowledges Prime Minister's Research Fellowship (PMRF), MHRD for financial support. R. K. acknowledges INSPIRE, DST for financial support. C. S.,
A. D. M., and Y. G. acknowledge support by DFG Grants No. MI 658/10-1 and No. MI 658/10-2 and by the GermanIsraeli Foundation Grant No. I-1505-303.10/2019. A. D. and Y.G. acknowledge ICTS, Novel Phases of the Quantum Matter Conference for initiation of collaboration. Y. G. acknowledges support by the Helmholtz International Fellow Award. K. W. and T. T. acknowledge support from the Elemental Strategy Initiative conducted by the MEXT, Japan and the CREST (JPMJCR15F3), JST.

S. K. S and R. K. contributed equally to this work.

* Corresponding author. anindya@iisc.ac.in

[1] X.-G. Wen, Int. J. Mod. Phys. B 06, 1711 (1992).

[2] B. Swingle and T. Senthil, Phys. Rev. B 86, 045117 (2012).

[3] J. Dubail, N. Read, and E. H. Rezayi, Phys. Rev. B 86, 245310 (2012).

[4] C. L Kane and M. P. A Fisher, Phys. Rev. Lett. 76, 3192 (1996).

[5] M. Banerjee, M. Heiblum, A. Rosenblatt, Y. Oreg, D. E. Feldman, A. Stern, and V. Umansky, Nature (London) 545, 75 (2017).

[6] M. Banerjee, M. Heiblum, V. Umansky, D. E. Feldman, Y. Oreg, and A. Stern, Nature (London) 559, 205 (2018).

[7] I. Protopopov, Y. Gefen, and A. Mirlin, Ann. Phys. (Amsterdam) 385, 287 (2017).

[8] C. Nosiglia, J. Park, B. Rosenow, and Y. Gefen, Phys. Rev. B 98, 115408 (2018).

[9] A. H MacDonald, Phys. Rev. Lett. 64, 220 (1990).

[10] C. L. Kane, M. P. A. Fisher, and J. Polchinski, Phys. Rev. Lett. 72, 4129 (1994).

[11] We assume generally that the coherence length is the shortest physical length scale governing edge transport. Then, equilibrated transport is always incoherent.

[12] Y. Cohen, Y. Ronen, W. Yang, D. Banitt, J. Park, M. Heiblum, A. D. Mirlin, Y. Gefen, and V. Umansky, Nat. Commun. 10, 1920 (2019).

[13] Z.-X. Hu, R. N Bhatt, X. Wan, and K. Yang, Phys. Rev. Lett. 107, 236806 (2011).

[14] G. Li, A. Luican-Mayer, D. Abanin, L. Levitov, and E. Y. Andrei, Nat. Commun. 4, 1744 (2013).

[15] S. K. Srivastav, M. R. Sahu, K. Watanabe, T. Taniguchi, S. Banerjee, and A. Das, Sci. Adv. 5, eaaw5798 (2019).

[16] S. Jezouin, F. Parmentier, A. Anthore, U. Gennser, A. Cavanna, Y. Jin, and F. Pierre, Science 342, 601 (2013).

[17] J. Li, C. Tan, S. Chen, Y. Zeng, T. Taniguchi, K. Watanabe, J. Hone, and C. Dean, Science 358, 648 (2017).

[18] C. Kumar, S. K. Srivastav, and A. Das, Phys. Rev. B 98, 155421 (2018).

[19] See Supplemental Material at http://link.aps.org/ supplemental/10.1103/PhysRevLett.126.216803 for details of sample characterization, measurements, additional data, and theoretical analysis, which includes Refs. [20-32].

[20] F. Pizzocchero, L. Gammelgaard, B. S. Jessen, J. M. Caridad, L. Wang, J. Hone, P. Bøggild, and T. J. Booth, Nat. Commun. 7, 11894 (2016). 
[21] A. Venugopal, J. Chan, X. Li, C. W. Magnuson, W. P. Kirk, L. Colombo, R. S. Ruoff, and E. M. Vogel, J. Appl. Phys. 109, 104511 (2011).

[22] C. Kumar, S. K. Srivastav, P. Adhikary, S. Banerjee, T. Das, and A. Das, Phys. Rev. B 98, 155408 (2018).

[23] M. Kuiri, S. K. Srivastav, S. Ray, K. Watanabe, T. Taniguchi, T. Das, and A. Das, Phys. Rev. B 103, 115419 (2021).

[24] B.-R. Choi, A. E Hansen, T. Kontos, C. Hoffmann, S. Oberholzer, W. Belzig, C. Schönenberger, T. Akazaki, and H. Takayanagi, Phys. Rev. B 72, 024501 (2005).

[25] C. Spånslätt, J. Park, Y. Gefen, and A. D. Mirlin, Phys. Rev. Lett. 123, 137701 (2019).

[26] A. O. Slobodeniuk, I. P. Levkivskyi, and E. V. Sukhorukov, Phys. Rev. B 88, 165307 (2013).

[27] P. Maher et al., Science 345, 61 (2014).

[28] X.-G. Wen, Adv. Phys. 44, 405 (1995).

[29] C. L. Kane and M. P. A. Fisher, Phys. Rev. B 52, 17393 (1995); 51, 13449 (1995).

[30] J. E. Moore and X.-G. Wen, Phys. Rev. B 57, 10138 (1998).

[31] T. Martin, Noise in mesoscopic physics, in Proceedings of the Les Houches Summer School, Session LXXXI, edited by H. Bouchiat et al. (Elsevier, New York, 2005).

[32] A. Cappelli, M. Huerta, and G. R. Zemba, Nucl. Phys. B636, 568 (2002).

[33] Our regime is very different from that in Refs. [34-36] where energy relaxation was much faster than charge relaxation.

[34] H. le Sueur, C. Altimiras, U. Gennser, A. Cavanna, D. Mailly, and F. Pierre, Phys. Rev. Lett. 105, 056803 (2010).
[35] C. Altimiras, H. le Sueur, U. Gennser, A. Cavanna, D. Mailly, and F. Pierre, Phys. Rev. Lett. 105, 226804 (2010).

[36] A. Rosenblatt, S. Konyzheva, F. Lafont, N. Schiller, J. Park, K. Snizhko, M. Heiblum, Y. Oreg, and V. Umansky, Phys. Rev. Lett. 125, 256803 (2020).

[37] M. Büttiker, Phys. Rev. B 38, 9375 (1988).

[38] U. Sivan and Y. Imry, Phys. Rev. B 33, 551 (1986).

[39] P. Butcher, J. Phys. Condens. Matter 2, 4869 (1990).

[40] J.-H. Jiang and Y. Imry, C. R. Phys. 17, 1047 (2016).

[41] C. W. J. Beenakker and M. Büttiker, Phys. Rev. B 46, 1889 (1992).

[42] Y. M. Blanter and E. V. Sukhorukov, Phys. Rev. Lett. 84, 1280 (2000).

[43] E. Sivre, A. Anthore, F. Parmentier, A. Cavanna, U. Gennser, A. Ouerghi, Y. Jin, and F. Pierre, Nat. Phys. 14, 145 (2018).

[44] C. Déprez, L. Veyrat, H. Vignaud, G. Nayak, K. Watanabe, T. Taniguchi, F. Gay, H. Sellier, and B. Sacépé, arXiv: 2008.11222.

[45] Y. Ronen et al., Nat. Nanotechnol. 16, 563 (2021).

[46] C. Spånslätt, Y. Gefen, I. V. Gornyi, and D. G. Polyakov, arXiv:2105.04013.

[47] K. K. W. Ma and D. E. Feldman, Phys. Rev. B 99, 085309 (2019).

[48] S. H. Simon and B. Rosenow, Phys. Rev. Lett. 124, 126801 (2020).

[49] H. Asasi and M. Mulligan, Phys. Rev. B 102, 205104 (2020).

[50] J. Park, C. Spånslätt, Y. Gefen, and A. D. Mirlin, Phys. Rev. Lett. 125, 157702 (2020). 